\title{
The value of magnetic resonance imaging-ultrasound fusion targeted biopsies for clinical decision making among patients with previously negative transrectal ultrasound biopsy and persistent prostate-specific antigen elevation
}

\author{
Charlie J. Gillis ${ }^{1}$; Thomas M. Southall ${ }^{2}$; Rob Wilson ${ }^{2}$; Michelle Anderson ${ }^{3}$; Jennifer Young ${ }^{4}$; \\ Richard Hewitt ${ }^{4}$; Matthew Andrews ${ }^{4}$ \\ ${ }^{1}$ Department of Urology, Dalhousie University, Halifax, NS, Canada; ${ }^{2}$ Department of Surgery, Section of Urology, \\ University of Manitoba, Winnipeg, MB, Canada, ${ }^{3}$ Department of Radiology, Health Sciences Centre, St. John's, NL, \\ Canada; ${ }^{4}$ Department of Surgery, Division of Urology, Health Sciences Centre, St. John's, NL, Canada
}

Cite as: Gillis CJ, Southall TM, Wilson R, et al. The value of magnetic resonance imagingultrasound fusion targeted biopsies for clinical decision making among patients with previously negative transrectal ultrasound biopsy and persistent prostate-specific antigen elevation. Can Urol Assoc J 2022 January 27; Epub ahead of print. http://dx.doi.org/10.5489/cuaj.7509

Published online January 27, 2022

Corresponding author: Dr. Matthew Andrew, Department of Surgery, Division of Urology, Health Sciences Centre, St. John’s, NL, Canada; matthewandrews@munmed.ca

$* * *$

\section{Abstract}

Introduction: Targeted biopsy approaches have been shown to increase the detection of clinically significant prostate cancer (csPCa) within index prostate lesions. We report our initial experience with magnetic resonance imaging-ultrasound fusion biopsies (MRI-TB) in a population of men who had a previously negative transrectal ultrasound (TRUS) biopsy, persistent prostate-specific antigen (PSA) elevation, and ongoing suspicion of PCa. Patients were followed prospectively to assess for changes in clinical management following targeted biopsy. Methods: We prospectively followed the first 122 patients undergoing MRI-TB at our institution. All men had clinical suspicion of $\mathrm{PCa}$, prior negative TRUS biopsies, and persistent PSA elevation. A total of 177 index lesions were identified on multiparametric MRI and reviewed using the prostate imaging reporting and data system (PI-RADS) V2 scoring system. Lesions classified as PI-RADS $\geq 3$ received targeted biopsy. Biopsy-naive patients and those on active surveillance were excluded. The primary outcome was detection rate of csPCa, defined as International Society of Urological Pathology (ISUP) Grade Group (GG) $\geq 2$. Multivariate analysis was used to determine predictors of csPCa on fusion biopsy.

Results: Prior to fusion biopsy, patients had a mean of 17.9 \pm 8.6 negative core biopsies per patient and a median PSA of 9.5 (standard deviation [SD] 6.2) ng/nl. MRI-TB resulted in 
diagnosis of csPCa in 42/122 (34.4\%) patients. Clinically significant $\mathrm{PCa}$ was found in eight (13.1\%), 14 (21.9\%), and 25 (48.1\%) of PI-RADS 3, 4, and 5 lesions, respectively. The location of csPCa was within the peripheral zone (55.3\%), transitional zone (40.4\%), and central zone (8.5\%). Clinical outcomes of patients with newly diagnosed csPCa show $4.8 \%, 57.1 \%$, and $38.1 \%$ receiving active surveillance, radiation treatment, and radical prostatectomy, respectively. Predictors for csPCa were presence of PI-RADS 5 lesions, age, length of time from MRI to biopsy, and smaller prostate volumes.

Conclusions: MRI-TB yields high detection rates for csPCa in men with elusive PSA elevation and frequently guides a change in clinical management. Clinical decision-making based on MRI findings and PI-RADS lesion scores are best informed by an understanding of institutional reporting patterns.

\section{Introduction}

Traditional systematic transrectal ultrasound (TRUS) guided prostate biopsy has the unfortunate limitation of missing lesions that are present outside the range of normal sampling ${ }^{1}$. Falsenegative rates are as high as $30-45 \%$, resulting in diagnostic uncertainty and undertreatment ${ }^{2,3}$. There is now increasing recommendation to incorporate MRI in the typical workup of suspected localized prostate cancer ${ }^{4-6}$. Compared with targeted biopsy strategies incorporating MRI, standard TRUS biopsies have been shown to miss clinically significant prostate cancers, particularly when a patient is harboring disease in the anterior or periurethral portions of the gland $^{7-11}$. In the setting where patients have received a previously negative standard TRUS biopsy, there is robust evidence that an MRI-targeted biopsy strategy improves the detection of csPCa and decreases the incidence of clinically insignificant prostate cancer ${ }^{12,13}$. For biopsynegative patients, a subsequent MRI-targeted biopsy can result in detection of csPCa in 34\% of patients compared to $16 \%$ when using TRUS-biopsy alone ${ }^{14}$. This often results in a change in clinical management as a number of patients will require subsequent intervention in the form of surgery, radiation, or active surveillance.

An ideal diagnostic test is one that can reliably and thoroughly detect true incidence of disease, while minimizing detection of insignificant findings, and does so in a timely fashion with minimal interventional risk. Multiparametric MRI is a useful tool in the detection of prostate cancer, with an overall sensitivity of $91 \%$ and specificity of $37 \%$ in a mixed population of biopsy naïve and previous biopsy patients ${ }^{4}$. Sensitivity rates increase for the detection of higher grade prostate cancer, and MRI findings have been shown to correlate with prostate risk stratification ${ }^{15}$. MRI-TB offers the advantage and precision to specifically target a lesion, potentially catching prostate cancer in atypical areas which may be missed by routine TRUS biopsy. Repeat TRUS biopsy has limited value in this setting, with one series reporting the detection of csPCa to be as low as $7.7 \%{ }^{16}$. There is also diminished cancer detection with 
subsequent biopsies, and much of the cancer detected is low risk, at the cost of increased complications and potential overtreatment ${ }^{17}$.

The incorporation of MRI and fusion technology has allowed for more precise sampling of suspicious index lesions, reducing over-detection of disease by traditional methods and increasing the detection of prostate cancer that requires intervention ${ }^{8,18}$. However, there are concerning rates of inter-observer reliability using MRI when scans are re-read by high-volume tertiary centre radiologists; in addition, much of the evidence supporting MRI use has been drawn from large tertiary centers ${ }^{19}$. Evidence is limited in smaller, low volume centers. This study examines the clinical outcome of biopsy-negative men undergoing MRI-TB for suspicion of prostate cancer in a small academic setting.

\section{Methods}

\section{Patient selection}

This study received ethics approval by the Newfoundland and Labrador Health Research Ethics Board (HREB 20192982). This prospective study was performed between September 2018 and July 2020 following the inception of MRI-TB technique at a single tertiary care center in Atlantic Canada. Our cohort of men all had at least one previous systematic TRUS biopsy with ongoing clinical suspicion of prostate cancer via persistent PSA elevation. Patients then received a prostatic MRI followed by MRI-TB if an index lesion was found to be PIRADS 3 or greater. Patients were followed prospectively to identify any change in clinical management as result of their targeted biopsy findings. Exclusion criteria included patients with normal prostatic MRI, PIRADS < 3 lesions, and men receiving MRI-TB as part of active surveillance.

\section{Imaging acquisition and biopsy protocol}

Multi-parametric prostatic MRI was performed using either a $1.5 \mathrm{~T}$ or 3T MRI scanner (Siemens) and reported by one of 6 MRI subspecialty radiologists. Index lesions on MRI were reviewed using the PIRADS v2 scoring system, with scores ranging from 1-5 to indicate the likelihood of csPCa. MRI-TB was performed in patients with PIRADS 3 or greater index lesions. BkFusion US technology and MIM software was used to fuse the US and MRI imagery in conventional manner. 3D contouring of index lesions was performed by the interpreting radiologist. Biopsies were performed by one of eight urologists at the Health Sciences Centre in St. John's, Newfoundland and Labrador. Transrectal biopsies were performed under local periprostatic anesthetic with the patient in left lateral position. The exact number of biopsy cores per index lesion was at the discretion of the surgeon based upon US findings and clinical judgement. Core biopsy tissue was centrally reviewed by 2 genitourinary subspecialty trained pathologists prior to a consensus diagnosis. Clinically significant prostate cancer was defined as ISUP Grade Group score $\geq 2$. 


\section{Statistical analysis}

Descriptive statistics and frequencies were reported for all patients. Logistic regression was performed to predict risk of csPCa using the following variables: MRI to fusion days, biopsy to fusion days, prostate volume, PIRADS lesion, tumor location, tumor zone, PSA, PSA density, and age. Only those variables with a $p$ value $\leq 0.05$ on univariate analysis were entered into the multivariate model. PIRADS lesions and tumour location were included as bivariate variables in the multivariate analysis. All statistical analyses were performed by a biostatistician using IBM SPSS Statistics 25 .

\section{Results}

\section{Demographics}

The study included 122 patients with a median age of 65 years (range 44-80) (Table 1). Participants had a median PSA of $9.5 \mathrm{ng} / \mathrm{ml}$ (standard deviation [SD]: $6.2 \mathrm{ng} / \mathrm{ml}$ ), a median PSA density of $0.188 \mathrm{ng} / \mathrm{ml} / \mathrm{cc}$ (SD: $0.164 \mathrm{ng} / \mathrm{ml} / \mathrm{cc}$ ), and a median prostate volume on MRI of 59.9 $\mathrm{cm}^{3}$ (SD: $43.8 \mathrm{~cm}^{3}$ ). The median interval from mpMRI to fusion biopsy was 75 days (SD 131.9 days). Prior to fusion biopsy, patients had a mean of $17.9 \pm 8.6$ negative core biopsies per patient. During fusion biopsy, patients had a mean of $2.8 \pm 1.1$ core biopsies per index lesion.

\section{Diagnostic yield of MRI-TB}

MRI-TB resulted in the diagnosis of PCa in 54/122 (44.3\%) patients. CsPCa was found in 5 (7.8\%), 14 (21.2\%), 23 (44.2\%) of PIRADS 3, 4, and 5 lesions, respectively. CsPCa was found within the peripheral zone (54.8\%), transitional zone (40.5\%), and central zone (9.5\%) (Table 2). The location of csPCa lesions were within the anterior gland 23 (48.9\%), midgland 16 (34.0\%), and posterior gland $8(17.0 \%)$ (Table 2$)$.

\section{Clinical outcomes and location}

Clinical outcomes of patients with newly diagnosed csPCa show $4.8 \%, 57.1 \%$, and $38.1 \%$ undergoing active surveillance, radiation treatment, and radical prostatectomy, respectively (Table 3). Of the 122 patients who participated in the study, 40 patients (32.8\%) ultimately chose treatment in the form of surgery or radiation therapy following a diagnosis of csPCa.

\section{Multivariate analysis}

Results of univariate analysis are presented in Table 4 and multivariate logistic regression model in Table 5. Increasing PIRADS score was associated with csPCa, as patients with PIRADS 5 lesions were 3.7 times more likely to have csPca when compared to other lesions (PIRADS 3 or 4). Increasing age was associated with diagnosis of csPCa, while greater length of time from MRI to fusion and increased prostate volume were associated with a reduction in risk of csPCa. Lesion location and PSA density were not found to be significant. 


\section{Discussion}

In this population of TRUS biopsy-negative men who underwent MRI-TB for persistently elevated PSA, the detection rate of csPCa was found to be $34.4 \%$. This led to a substantial change in clinical management as most patients consequently chose to undergo radical prostatectomy or radiation therapy. A large percentage of csPCa was found in anterior lesions (48.9\%), suggesting that disease may be persistent in this area after an initial negative TRUS biopsy. These findings are consistent with established literature suggesting the benefit of MRITB in the biopsy-negative setting. In a multi-institutional cohort of 779 patients conducted by Sidana $\mathrm{et}^{20}{ }^{20}$, the csPCa detection rate by MRI-TB was found to be $26.3 \%$. In a systematic review and meta-analysis by Schoots et $\mathrm{al}^{8}$, MRI-TB in biopsy-negative patients outperformed TRUS with a relative sensitivity of 1.54 (95\% CI 1.05-2.26) for the detection of csPCa. Our data confirms the utility of MRI-TB to detect elusive disease and suggests that a significant number of these patients (32.8\%) will eventually require treatment.

We found csPCa detection rates of 7.8\%, 21.2\%, and 44.2\% for PIRADS 3, 4, and 5 lesions respectively, which could be considered low when initially comparing to contemporary series. The EAU reports a working range of detection by PIRADS score, with rates of 4-27\% (PIRADS 3), 32-60\% (PIRADS 4), and 67-83\% (PIRADS 5) ${ }^{21}$. A large meta-analysis performed by Park et al reported detection rates as $17 \%, 46 \%$, and $75 \%$, for PIRADS 3,4 , and 5 respectively ${ }^{22}$. However, such series involve a heterogenous mix of biopsy-naïve and previous biopsy patients, and thus differ from our population composed solely of previous biopsynegative patients. In fact, our results are similar to Sathianathen et al who reported a lower detection rate of csPCa -- 3\%, 16\%, and 58\% for PIRADS 3, 4, and 5, respectively -- in previous biopsy patients when compared to biopsy-naïve patients or patients on active surveillance ${ }^{23}$. In reality, the lower detection rates per PIRADS 4 and 5 lesions identified in our study is likely multifactorial and portrays the trials and tribulations of a new biopsy technique, new contouring software, and associated learning curves.

We confirmed that PIRADS 5 lesions were associated with higher detection rates of csPCa by targeted biopsy with an OR of 3.7, in comparison to either PIRADS 3 or 4 lesions. However, neither PIRADS 3 nor 4 lesions were significantly associated with higher detection rates in our study; a discovery plausibly reflective of our sample size, learning curve, and/or institutional reporting patterns. Previous publications indicated that when radiologists in tertiary centers re-evaluated prostate MRIs performed at a regional center, there was disagreement in as many as $54 \%$ of reports, with second reads resulting in an improved PPV and NPV ${ }^{19}$. Thus, there is concern for the application of MRI ubiquitously in settings with limited access and detection rates likely more pragmatic as in our results. Outside of a high-volume centre, Kohestani et al ${ }^{24}$ showed that there was only moderate agreement $(\kappa=0.41)$ between readers. These findings and limitations of MRI usage, as well as local reporting rates, should be taken into account when counseling a patient with clinical suspicion of prostate cancer. 
For patients undergoing repeat biopsy for persistent suspicion of csPCa, MRI-TB consistently outperforms TRUS, yet there remains concern for disease that is potentially also missed on MRI-TB. The quantity of missed disease has been widely reported between $5.6-$ $15 \%,{ }^{23,26,27}$ and thus the decision to perform concurrent systematic biopsy alongside MRI-TB should be made on the basis of individualized prostate cancer risk and risk tolerance. Current recommendations are based on a follow-up analysis of the FUTURE trial ${ }^{14}$ which looked at patients receiving both MRI-TB and standard biopsies. In a core-by-core analysis, standard TRUS biopsy resulted in an additional csPCa diagnosis of $1.3 \%$ that would have been missed if exclusively MRI-TB was performed. This is balanced against the potential for increased adverse effects with MRI-TB plus standard TRUS biopsy, as more biopsy cores infers a longer procedure time, greater patient discomfort, and possibly increased risk of bleeding. Indeed, Arsov et $\mathrm{al}^{25}$ found that one csPCa diagnosis was the result of 19 MRI-TB cores, compared to 55 when using standard TRUS biopsy.

Our study has several limitations. First, patients undergoing fusion biopsy only had MRITB completed at the time of the procedure limiting our ability to directly compare detection rates to a standard TRUS biopsy method. Another limitation includes the extended time interval between mpMRI and fusion biopsy (Table 1). The large deviation in time is representative of the challenges faced by patients in Newfoundland. The large geographic area covered by our tertiary care centre, companied with a small number of MRI scanners, and unpredictable weather conditions often result in appointment delays. Initial technical issues were also experienced with contouring software which led to a transient delay in performance of targeted biopsies until resolved. Greater duration from imaging to MRI-TB can conceptually result in lesion growth during that time, increasing the positive detection rate upon targeted biopsy particularly in smaller volume prostates. Albeit, this could also influence contour positioning if prostate gland geometry had altered in the interim. Thirdly, data collection for this study began with inception of MRI-US fusion biopsies at our centre, possibly limiting the applicability of early biopsies completed during the initial learning curve experienced by both radiology and urology staff. Fourthly, each radiologist individually reported their MRI findings without a central review panel, thus creating inter-reader variability. Finally, when performing MRI-TB the previously mapped fusion image may undergo compression by the endorectal ultrasound probe affecting the precision of biopsy particularly when aiming for smaller lesions. The device used in this study does not accommodate for this gland compression, whereas other series may have varied results based on devices which do accommodate.

Despite these limitations, we believe our findings are still valid and reflect the real-world utility of prostate fusion biopsies in a pragmatic setting. We believe this data will be of interest to community urology or small academic centers contemplating or beginning MRI-US fusion biopsy techniques. Few smaller centers have published similar series, despite the importance of quality assurance to confirm that local data is in keeping with large center studies. 


\section{Conclusions}

In patients with previous negative TRUS biopsies, clinically significant prostate cancer was detected by MRI fusion biopsies in $34.4 \%$ of cases, leading to a change in clinical management for many of these patients. It is important to establish institutional detection rates of PIRADS lesions as this can significantly affect the clinical management of prostate cancer patients. 


\section{References}

1. Djavan B, Zlotta A, Remzi M, et al. OPTIMAL PREDICTORS OF PROSTATE CANCER ON REPEAT PROSTATE BIOPSY: A PROSPECTIVE STUDY OF 1,051 MEN. J Urol. 2000;163(4):1144-1149. doi:10.1016/S0022-5347(05)67711-7

2. Brown LC, Ahmed HU, Faria R, et al. Multiparametric MRI to improve detection of prostate cancer compared with transrectal ultrasound-guided prostate biopsy alone: the PROMIS study. Health Technol Assess. 2018;22(39):1-176. doi:10.3310/hta22390

3. Taira AV, Merrick GS, Galbreath RW, et al. Performance of transperineal templateguided mapping biopsy in detecting prostate cancer in the initial and repeat biopsy setting. Prostate Cancer Prostatic Dis. 2010;13(1):71-77. doi:10.1038/pcan.2009.42

4. Drost F-JH, Osses DF, Nieboer D, Steyerberg EW, Bangma CH, Roobol MJ. Prostate MRI, with or without MRI-targeted biopsy, and systematic biopsy for detecting prostate cancer. Cochrane Database Syst Rev. 2019;(4). doi:10.1002/14651858.CD012663.pub2

5. Mottet N, van den Bergh RCN, Briers E, et al. EAU-EANM-ESTRO-ESUR-SIOG Guidelines on Prostate Cancer-2020 Update. Part 1: Screening, Diagnosis, and Local Treatment with Curative Intent. Eur Urol. 2021;79(2):243-262. doi:10.1016/j.eururo.2020.09.042

6. Porpiglia F, Manfredi M, Mele F, et al. Diagnostic Pathway with Multiparametric Magnetic Resonance Imaging Versus Standard Pathway: Results from a Randomized Prospective Study in Biopsy-naïve Patients with Suspected Prostate Cancer. Eur Urol. 2017;72(2):282-288. doi:10.1016/j.eururo.2016.08.041

7. Mendhiratta N, Meng X, Rosenkrantz AB, et al. Prebiopsy MRI and MRI-ultrasound Fusion-targeted Prostate Biopsy in Men With Previous Negative Biopsies: Impact on Repeat Biopsy Strategies. Urology. 2015;86(6):1192-1199. doi:10.1016/j.urology.2015.07.038

8. Schoots IG, Roobol MJ, Nieboer D, Bangma CH, Steyerberg EW, Hunink MGM. Magnetic Resonance Imaging-targeted Biopsy May Enhance the Diagnostic Accuracy of Significant Prostate Cancer Detection Compared to Standard Transrectal Ultrasoundguided Biopsy: A Systematic Review and Meta-analysis. Eur Urol. 2015;68(3):438-450. doi:10.1016/j.eururo.2014.11.037

9. Siddiqui MM, Rais-Bahrami S, Truong H, et al. Magnetic Resonance Imaging/Ultrasound-Fusion Biopsy Significantly Upgrades Prostate Cancer Versus Systematic 12-core Transrectal Ultrasound Biopsy. Eur Urol. 2013;64(5):713-719. doi:10.1016/j.eururo.2013.05.059

10. Vourganti S, Rastinehad A, Yerram NK, et al. Multiparametric Magnetic Resonance Imaging and Ultrasound Fusion Biopsy Detect Prostate Cancer in Patients with Prior Negative Transrectal Ultrasound Biopsies. J Urol. 2012;188(6):2152-2157. doi:10.1016/j.juro.2012.08.025

11. van Hove A, Savoie P-H, Maurin C, et al. Comparison of image-guided targeted biopsies versus systematic randomized biopsies in the detection of prostate cancer: a systematic literature review of well-designed studies. World J Urol. 2014;32(4):847-858. doi:10.1007/s00345-014-1332-3

12. Capitanio U, Pfister D, Emberton M. Repeat Prostate Biopsy: Rationale, Indications, and Strategies. Eur Urol Focus. 2015;1(2):127-136. doi:10.1016/j.euf.2015.05.002 
13. Truong M, Frye TP. Magnetic resonance imaging detection of prostate cancer in men with previous negative prostate biopsy. Transl Androl Urol. 2017;6(3):42431-42431.

14. Exterkate L, Wegelin O, Barentsz JO, et al. Is There Still a Need for Repeated Systematic Biopsies in Patients with Previous Negative Biopsies in the Era of Magnetic Resonance Imaging-targeted Biopsies of the Prostate? Eur Urol Oncol. 2020;3(2):216-223. doi:10.1016/j.euo.2019.06.005

15. Rastinehad AR, Baccala AA, Chung PH, et al. D'Amico risk stratification correlates with degree of suspicion of prostate cancer on multiparametric magnetic resonance imaging. $J$ Urol. 2011;185(3):815-820. doi:10.1016/j.juro.2010.10.076

16. Yoo S, Park J, Cho SY, et al. Predictors for the detection of prostate cancer and clinically significant prostate cancer using TRUS-guided biopsy in patients with negative initial biopsy results. World J Urol. 2018;36(7):1047-1053. doi:10.1007/s00345-018-2239-1

17. Djavan B, Ravery V, Zlotta A, et al. PROSPECTIVE EVALUATION OF PROSTATE CANCER DETECTED ON BIOPSIES 1, 2, 3 AND 4: WHEN SHOULD WE STOP? $J$ Urol. 2001;166(5):1679-1683. doi:10.1016/S0022-5347(05)65652-2

18. Sonn GA, Chang E, Natarajan S, et al. Value of Targeted Prostate Biopsy Using Magnetic Resonance-Ultrasound Fusion in Men with Prior Negative Biopsy and Elevated Prostate-specific Antigen. Eur Urol. 2014;65(4):809-815. doi:10.1016/j.eururo.2013.03.025

19. Hansen NL, Koo BC, Gallagher FA, et al. Comparison of initial and tertiary centre second opinion reads of multiparametric magnetic resonance imaging of the prostate prior to repeat biopsy. Eur Radiol. 2017;27(6):2259-2266. doi:10.1007/s00330-0164635-5

20. Sidana A, Watson MJ, George AK, et al. Fusion prostate biopsy outperforms 12-core systematic prostate biopsy in patients with prior negative systematic biopsy: A multiinstitutional analysis. Urol Oncol Semin Orig Investig. 2018;36(7):341.e1-341.e7. doi:10.1016/j.urolonc.2018.04.002

21. Israël B, Leest M van der, Sedelaar M, Padhani AR, Zámecnik P, Barentsz JO. Multiparametric Magnetic Resonance Imaging for the Detection of Clinically Significant Prostate Cancer: What Urologists Need to Know. Part 2: Interpretation. Eur Urol. 2020;77(4):469-480. doi:10.1016/j.eururo.2019.10.024

22. Park KJ, Choi SH, Lee JS, Kim JK, Kim M, Jeong IG. Risk Stratification of Prostate Cancer According to PI-RADS ${ }^{\circledR}$ Version 2 Categories: Meta-Analysis for Prospective Studies. J Urol. 2020;204(6):1141-1149. doi:10.1097/JU.0000000000001306

23. Sathianathen NJ, Link to external site this link will open in a new window, Konety BR, et al. Which scores need a core? An evaluation of MR-targeted biopsy yield by PIRADS score across different biopsy indications. Prostate Cancer Prostatic Dis. 2018;21(4):573578. doi:http://dx.doi.org.qe2a-proxy.mun.ca/10.1038/s41391-018-0065-6

24. Kohestani K, Wallström J, Dehlfors N, et al. Performance and inter-observer variability of prostate MRI (PI-RADS version 2) outside high-volume centres. Scand J Urol. 2019;53(5):304-311. doi:10.1080/21681805.2019.1675757

25. Arsov C, Rabenalt R, Blondin D, et al. Prospective Randomized Trial Comparing Magnetic Resonance Imaging (MRI)-guided In-bore Biopsy to MRI-ultrasound Fusion and Transrectal Ultrasound-guided Prostate Biopsy in Patients with Prior Negative Biopsies. Eur Urol. 2015;68(4):713-720. doi:10.1016/j.eururo.2015.06.008 
Figures and Tables

\begin{tabular}{|l|l|}
\hline $\begin{array}{l}\text { Table 1. Demographics in patients with persistent PSA elevation and previously negative } \\
\text { TRUS prostate biopsy now undergoing MRI-US fusion prostate biopsy (n=122) }\end{array}$ \\
\hline & Mean/median (SD) \\
\hline Age (years) & $64.4 / 65.0(7.1)$ \\
\hline Most recent PSA $(\mathrm{ng} / \mathrm{ml})$ & $11.5 / 9.5(6.2)$ \\
\hline PSA density $(\mathrm{ng} / \mathrm{ml} / \mathrm{cc})$ & $0.217 / 0.188(0.164)$ \\
\hline Prostate volume $\left(\mathrm{cm}^{3}\right)$ & $77.3 / 59.9(43.8)$ \\
\hline Interval between MRI and fusion biopsy (days) & $128.33 / 75.0(131.9)$ \\
\hline Number of cores per lesion & $2.8 / 3.0(1.1)$ \\
\hline Number of cores per patient prior to fusion Bx & $17.9 / 12(8.6)$ \\
\hline
\end{tabular}

Bx: biopsy; MRI: magnetic resonance imaging; PSA: prostate-specific antigen; TRUS:

transrectal ultrasound; US: ultrasound.

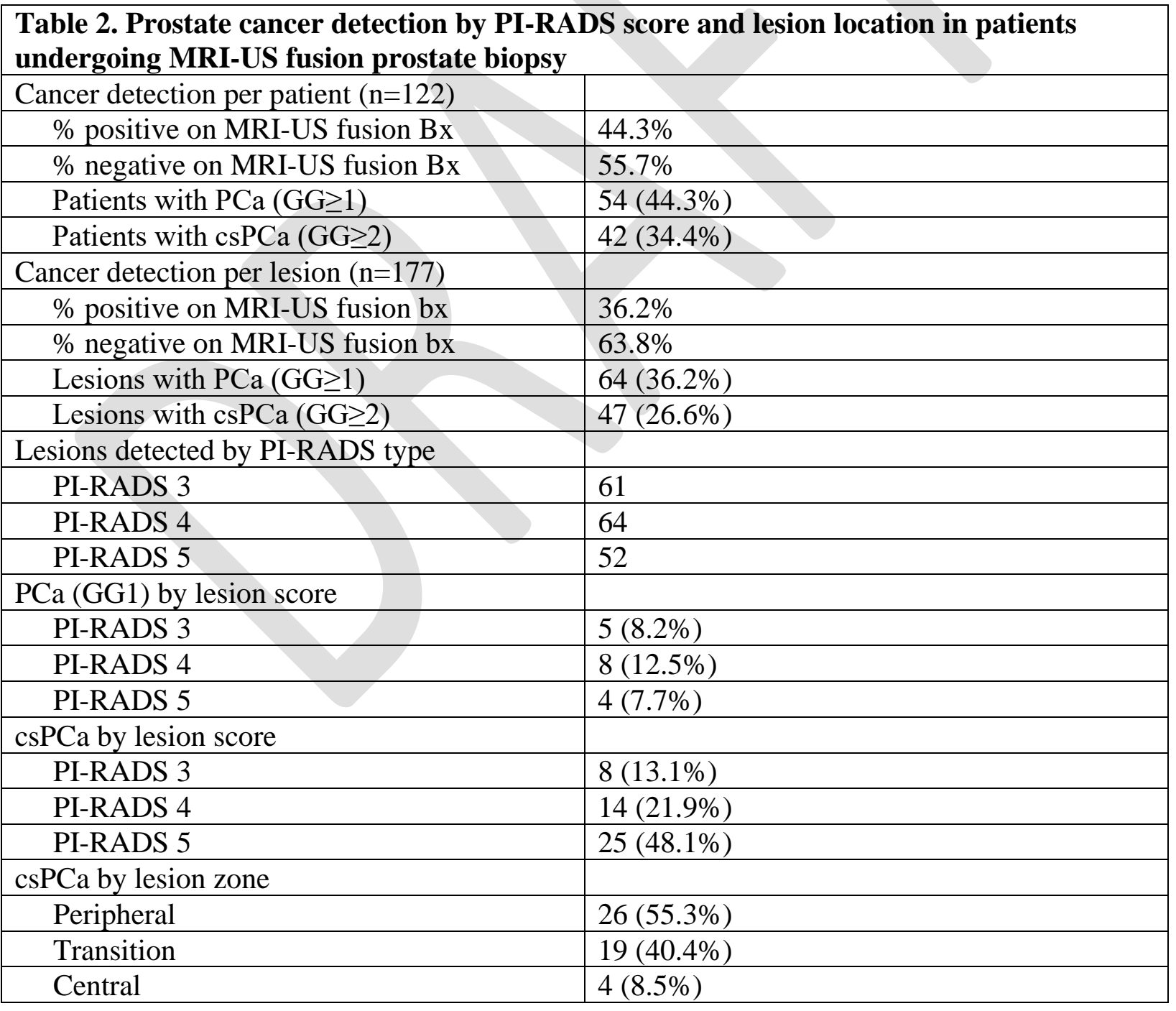




\begin{tabular}{|l|l|}
\hline csPCa by lesion location & \\
\hline Anterior & $23(48.9 \%)$ \\
\hline Midgland & $16(34.0 \%)$ \\
\hline Posterior & $8(17.0 \%)$ \\
\hline
\end{tabular}

Bx: biopsy; csPCa: clinically significant prostate cancer; GG: grade group; MRI: magnetic resonance imaging; PCa: prostate cancer; PI-RADS: prostate imaging reporting and data system. US: ultrasound.

\begin{tabular}{|l|l|}
\hline \multicolumn{2}{|l|}{ Table 3. Clinical outcomes of patients following detection of csPCa on MRI-TB } \\
\hline Patients with csPCa $(\mathrm{GG} \geq 2)$ & $42 / 122(34.4 \%)$ \\
\hline Resulting treatment received by patients with csPCa: & \\
\hline Active surveillance & $2(4.8 \%)$ \\
\hline Radiation treatment & $24(57.1 \%)$ \\
\hline Radical prostatectomy & $16(38.1 \%)$ \\
\hline
\end{tabular}
csPCa: clinically significant prostate cancer; GG: grade group; MRI-TB: magnetic resonance imaging-targeted biopsy.

\begin{tabular}{|c|c|c|c|c|}
\hline \multicolumn{5}{|c|}{$\begin{array}{l}\text { Table 4. Univariate analysis of factors predicting the detection of csPCa by MRI-US } \\
\text { fusion biopsy in patients with prior negative TRUS biopsy and persistent PSA elevation } \\
(\mathrm{n}=122)\end{array}$} \\
\hline Feature & $\begin{array}{l}\text { Prostate } \\
\text { cancer, } \\
\text { n }(\%)\end{array}$ & $\begin{array}{l}\text { No prostate } \\
\text { cancer, } \\
\text { n }(\%)\end{array}$ & $\begin{array}{l}\text { Odds ratio } \\
(95 \% \mathrm{CI})\end{array}$ & $\mathbf{p}$ \\
\hline $\begin{array}{l}\text { mpMRI to fusion } \\
\text { (days) }\end{array}$ & & & $0.995(0.992-0.999)$ & $0.012^{*}$ \\
\hline $\begin{array}{l}\text { Biopsy to fusion } \\
\text { biopsy (days) }\end{array}$ & & & $1.000(0.999-1.000)$ & 0.556 \\
\hline $\begin{array}{l}\text { Prostate volume on } \\
\text { MRI }\end{array}$ & & & $0.981(0.971-0.991)$ & $0.000^{*}$ \\
\hline \multicolumn{5}{|l|}{ PI-RADs lesions } \\
\hline 3 & $8(13.1)$ & $53(86.9)$ & $0.298(0.129-0.688)$ & $0.005^{*}$ \\
\hline 4 & $14(21.9)$ & $50(78.1)$ & $0.679(0.331-1.392)$ & 0.290 \\
\hline 5 & $25(48.1)$ & $27(51.9)$ & $4.335(2.126-8.841)$ & $0.000^{*}$ \\
\hline \multicolumn{5}{|l|}{ Index tumor location } \\
\hline Mid-gland & $16(18.8)$ & $69(81.2)$ & $0.456(0.228-0.914)$ & $0.027^{*}$ \\
\hline Anterior & $23(46.0)$ & $27(54.0)$ & $3.656(1.794-7.449)$ & $0.000^{*}$ \\
\hline Posterior & $8(19.0)$ & $34(81.0)$ & $0.579(0.246-1.362)$ & 0.211 \\
\hline \multicolumn{5}{|l|}{ Index tumor zone } \\
\hline Central & $4(26.7)$ & $11(73.3)$ & $1.006(0.3304-3.329)$ & 0.992 \\
\hline Transitional & $17(20.7)$ & $65(79.3)$ & $0.567(0.285-1.127)$ & 0.105 \\
\hline
\end{tabular}




\begin{tabular}{|l|l|l|l|l|}
\hline \multicolumn{1}{|c|}{ Peripheral } & $26(32.5)$ & $54(67.5)$ & $1.743(0.889-3.414)$ & 0.106 \\
\hline PSA & & & $1.105(1.003-1.108)$ & $0.038^{*}$ \\
\hline PSA sensity & & & $68.33(6.990-668.032)$ & $0.000^{*}$ \\
\hline Age & & & $1.061(1.008-1.118)$ & $0.024^{*}$ \\
\hline
\end{tabular}

"Statistically significant $(\mathrm{p}<0.05)$. CI: confidence interval; csPCa: clinically significant prostate cancer; MRI: magnetic resonance imaging; PCa: prostate cancer; PI-RADS: prostate imaging reporting and data system; PSA: prostate-specific antigen; TRUS: transrectal ultrasound; US: ultrasound.

\begin{tabular}{|c|c|c|}
\hline \multicolumn{3}{|c|}{$\begin{array}{l}\text { Table 5. Multivariate logistic regression model for the prediction of csPCa on MRI- } \\
\text { fusion biopsy in men with prior negative TRUS biopsy and persistent PSA elevation } \\
(n=122)\end{array}$} \\
\hline Feature & Odds ratio (95\% CI) & $\mathbf{p}$ \\
\hline mpMRI to fusion (days) & $0.996(0.992-1.000)$ & $0.038^{*}$ \\
\hline Prostate volume on MRI & $0.971(0.952-0.990)$ & $0.003^{*}$ \\
\hline PI-RADs lesions $^{1}$ & ( & 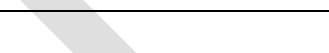 \\
\hline 3 & $1.007(0.332-3.055)$ & 0.990 \\
\hline 5 & $3.698(1.411-9.691)$ & $0.008^{*}$ \\
\hline \multicolumn{3}{|l|}{ Index tumor location $^{2}$} \\
\hline Mid-gland & $0.910(0.309-2.679)$ & 0.865 \\
\hline Anterior & $2.665(0.850-8.354)$ & 0.093 \\
\hline PSA & $1.021(0.905-1.152)$ & 0.734 \\
\hline PSA density & $0.347(0.002-57.831)$ & 0.685 \\
\hline 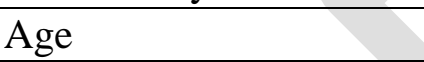 & $1.078(1.006-1.156)$ & $0.034^{*}$ \\
\hline
\end{tabular}

${ }^{1}$ Reference variable: Other PI-RADS lesions. ${ }^{2}$ Reference variable: Other lesion location.

"Statistically significant $(\mathrm{p}<0.05)$. CI: confidence interval; csPCa: clinically significant prostate cancer; mp: multiparametric; MRI: magnetic resonance imaging; PCa: prostate cancer; PIRADS: prostate imaging reporting and data system; PSA: prostate-specific antigen; TRUS: transrectal ultrasound; US: ultrasound. 